\title{
Retos de la formación médica de grado
}

Jorge L. Palés ${ }^{1,2}$ y Felipe Rodríguez de Castro ${ }^{1,3}$

1. Fundación Educación Médica.

2. Facultad de Medicina de la Universidad de Barcelona.

3. Facultad de Medicina de la Universidad de las Palmas

de Gran Canaria.

\section{INTRODUCCIÓN}

Las Facultades de Medicina españolas han iniciado un nuevo proceso de reforma curricular en el marco de la implantación del Espacio Europeo de Educación Superior (EEES). Este proceso constituye, sin duda, una nueva oportunidad, quizás la última en mucho tiempo, para llevar a cabo de una vez por todas la reforma en profundidad que nuestra formación de grado requiere. Los retos que se nos plantean son muchos y nada fáciles de afrontar. En este artículo vamos a discutir cuáles son estos retos y cómo deberíamos alcanzarlos. Para saber hacia dónde hemos de ir es imprescindible saber de dónde partimos y sobre todo dónde estamos. Por ello dividiremos nuestra exposición en tres períodos: el primero, que engloba el siglo XX desde 1930 a 1990, es un período en el que a pesar de su dilatada extensión apenas se producen cambios substanciales en nuestra formación de grado; el segundo, que se extiende desde finales del siglo XX a los principios del siglo actual (concretamente desde 1990 al 2003), abarca nuestro presente actual; y el tercero a partir del 2004 , en el que comienzan a surgir los retos que el futuro nos depara.

\section{EL SIGLO XX}

\section{De 1929 a 1990}

En este período la formación médica de grado en nuestro país no experimenta importantes modificaciones ni cambios radicales y en aquellos limitados casos en que se producen, éstos son aislados u obedecen a iniciativas individuales que en un plazo más o menos corto acaban por fracasar. Sólo se suceden cambios de planes de estudios caracterizados por modificaciones esencialmente de tipo estructural. De hecho, no se produce una reforma de cierta envergadura hasta 1990 que, por desgracia, como analizaremos posteriormente, no es bien aprovechada.

Sin embargo, este período no puede considerarse totalmente plano desde el punto de vista educativo y en él se pueden distinguir algunos hechos de cierta relevancia. En 1929 el fisiólogo Juan Negrín ${ }^{1}$ reorganiza el currículum preclínico en consonancia con los avances científicos de la época. Poco después, en 1933, surge la experiencia de la primera Universitat Autònoma de Barcelona. En ella, y por lo que hace referencia a la enseñanza de la Medicina, se proponen algunas reformas interesantes, como la desaparición de la enseñanza masificada y la supresión de las clases magistrales y su substitución por el método inductivo u objetivo o de autoaprendizaje, con observación directa del hecho biológico. La asistencia a las clases prácticas se hace indispensable, así como el seguimiento de historias clínicas y la presencia en intervenciones quirúrgicas y en necropsias. Se intenta, además, paliar la pobreza de medios materiales y de recursos humanos. La experiencia se ve truncada por nuestra guerra civil. ${ }^{2}$

Hasta la década de los sesenta, el modelo de los años treinta se mantiene prácticamente igual. En 1962 y en 1966, otro fisiólogo eminente, el profesor Antonio Gallego, publica en el Journal of Medical Education sendos artículos, en los que realiza un diagnóstico certero de la situación de la educación médica en nuestro país, analizando la realidad de la época y las tendencias de la Educación Médica en el mundo $^{3,4}$. En 1967, el mismo profesor Gallego, que en 1975 fundaría la Sociedad Española de Educación Médica (SEDEM), diseña un nuevo currículum, considerado como revolucionario para aquel momento ${ }^{5}$. Dicha reforma pretendía limitar el número de estudiantes que ingresaban en las Facultades de Medicina, preconizaba la integración de contenidos, incrementaba la enseñanza práctica, reducía la excesiva enseñanza de teórica y promovía la evaluación continua de los estudiantes. Cuando esta reforma se implementa, los estudiantes y los profesores consideran que se les exige demasiado y la experiencia dura sólo tres años. Se vuelve a la enseñanza tradicional y se suceden distintos planes de estudio con pocas diferencias entre ellos y sin introducirse cambios significativos en la metodología docente. Pero 
quizás uno de los aspectos más graves es que nuevamente el acceso de estudiantes a las Facultades de Medicina vuelve a ser totalmente libre. Esto produce un progresivo deterioro de la Educación Médica de grado y una sobresaturación de las Facultades de Medicina de nuestro país, llegándose a cifras de 22.564 estudiantes de nuevo ingreso en las 23 facultades existentes en 1977 y a un total de 70.232 estudiantes en todo el país. Sólo a título comparativo, aquel mismo año había en las 120 Facultades de los EE.UU. un total de 16.000 estudiantes de primer año1. Las cifras por sí solas son bastante elocuentes. En 1968 aparecen nuevas experiencias en las Facultades de Medicina de las Universidades Autónomas de Madrid y Barcelona, de reciente creación. En estas Facultades se introduce el numerus clausus y ciertos cambios curriculares, pero en el resto del país el caos sigue siendo la norma.

En 1977 se autoriza, por fin, de forma legal y para todas las Facultades de Medicina, el numerus clausus. De esta forma, las Facultades de Medicina limitan, no sin problemas ni presiones en contra, el número de estudiantes a ingresar, con reducciones significativas, aunque no del todo suficientes. La limitación permite cierta mejora de la enseñanza y el desarrollo de algunas experiencias innovadoras pero de corto alcance y duración.

En 1980 se crea una nueva Facultad de Medicina en la Universidad de Alicante. Bajo la dirección de los profesores Belmonte y García Barbero, se introduce un nuevo currículum basado en sistemas, con un importante nivel de integración de contenidos, con una clara orientación a la atención primaria de salud y bajo la dirección de una Unidad o Departamento de Educación Médica, el primero que se crea en España. La experiencia, única en el país y conocida como el "modelo Alicante", supone el cambio más innovador en la Educación Médica de grado en España durante mucho tiempo. Dura una serie de años, en los cuales sus impulsores tienen a su cargo la dirección de la Facultad. Sin embargo, al cambiar el equipo de gobierno la experiencia desaparece por las resistencias de determinados grupos de profesores.

A pesar del numerus clausus, hasta 1990 la situación global de la enseñanza en las Facultades de Medicina no mejora de forma substancial, ya que en los cursos superiores persisten grandes contingentes de estudiantes que imposibilitan una enseñanza de una mínima calidad. Se mantiene vigente en todas las Facultades de
Medicina españolas el plan de $1974^{7}$, totalmente monolítico, que perdura hasta 1990. Sin embargo, en estos años, aparece un hecho de capital importancia en la Educación Médica de nuestro país. En concreto, en el año 1978, se promulga un Real Decreto por el que se regulaba la formación médica especializada en unidades docentes acreditadas y se ponía en marcha el Sistema Nacional de Médicos Internos y Residentes (MIR). En esas fechas se constituyen también las primeras Comisiones Nacionales. Quedaba así derogado el decreto anteriormente vigente, de 1959, por el que se había regido la especialización médica -basada en maestros y escuelas profesionales, con primacía de la enseñanza teórica y con una actividad clínica voluntaria-, y la correspondiente titulación oficial durante casi veinte años. Poco tiempo después, en 1984, aparecía el RD 127/1984 por el que quedaban aún mejor establecidas las características que debía reunir la formación médica especializada y el procedimiento a seguir para obtener el título de médico especialista ${ }^{8,9}$. Se puede afirmar sin lugar a dudas que la implantación del sistema MIR, con responsabilidad progresiva supervisada del médico en formación, es el avance más importante en la Educación Médica española. Este sistema de formación especializada ha sido objeto de admiración en muchos países europeos y ha contribuido decisivamente a que la Educación Médica postgraduada fuera, desde el punto de vista cualitativo, la mejor de las tres fases del continuum educativo médico. Sin embargo, uno de sus principales defectos es el examen de acceso al mismo. $\mathrm{Al}$ ser mayoritariamente de tipo cognitivo, esta prueba ha condicionado e hipotecado gravemente hasta nuestros días los estudios de grado y las posibilidades de llevar a cabo una reforma en profundidad de los mismos ${ }^{10}$.

Durante este mismo período de tiempo, fuera de nuestras fronteras, se empiezan a producir cambios y avances muy importantes en la Educación Médica cuyas repercusiones en nuestro país se van a hacer esperar todavía mucho tiempo. Los principios de estos cambios están contenidos en diferentes documentos como la Declaración de Edimburgo (1988) ${ }^{11}$, en la que se tratan las prioridades de la Educación Médica, sus estrategias, los recursos humanos y financieros necesarios, las políticas de admisión a las Facultades de Medicina, la relación entre las diferentes fases de la enseñanza de la Medicina -pregrado, postgrado y formación médica continuada-, y la integración de esta enseñanza en el sistema sanitario. A la Declaración de Edimburgo le sigue la Iniciativa de 
Lisboa (OMS, 1988) ${ }^{12}$. En esta capital europea se reúnen a instancias de la OMS, los ministros de Educación y de Sanidad y delegados de 25 países europeos, entre ellos España. Esta iniciativa acepta los principios de la Declaración de Edimburgo y propone la realización de programas de cooperación internacional al objeto de reorientar la educación médica. Posteriormente, en 1993, se celebra la cumbre mundial de Educación Médica (World Summit on Medical Education (1994) ${ }^{13}$ y, en 1995, la OMS define las nuevas estrategias educativas en la formación de los profesionales de la salud, asignándoles nuevas funciones en su actuación como agentes sociales de la salud (Boelen, 1995) ${ }^{14,15}$. Otros documentos que también deben ser destacados en este sentido son las recomendaciones del General Medical Council (GMC) británico (Tomorrow's doctors, 1993) ${ }^{16,17}$ para la enseñanza de la Medicina en el Reino Unido. En el año 2000, la World Federation for Medical Education (WFME) define por primera vez los estándares internacionales para la Educación Médica de pregrado, que abundan en los principios anteriormente expresados y establecen claramente las condiciones que deberían reunir todas las Facultades para mejorar su función docente ${ }^{18}$.

Todas estas iniciativas determinan que en este período de tiempo cristalicen en muchas facultades -aunque, por desgracia, no en las españolas- el cambio de la enseñanza de la Medicina, cuyas claves pueden resumirse en: ${ }^{19}$

a) La introducción de nuevos métodos pedagógicos centrados en el estudiante y en su posición activa en el aprendizaje.

b) El desarrollo e implementación de instrumentos para la enseñanza/aprendizaje y para la evaluación, poniendo énfasis, además de en los conocimientos, en la enseñanza y en la evaluación de las habilidades clínicas y de las actitudes o pautas de comportamientos incluidas en los nuevos papeles asignados a los profesionales de la medicina.

c) El fomento de la enseñanza/aprendizaje orientado hacia los resultados (outcome- based medical education) que se desean obtener: implícita en esta orientación se halla el concepto de responsabilidad social inherente a la actividad educativa, como materia de interés público.

d) La evaluación del proceso, de la estructura y del resultado.

e) El desarrollo del profesorado como profesionales de la Educación Médica y la necesidad imperiosa de la valoración de la función docente en su selección.

f) El fomento de la investigación en Educación Médica y de la enseñanza de la Medicina basada en la mejor evidencia. De forma paralela a la medicina basada en la evidencia, se postula que la aplicación de cambios e innovaciones para dotar de la máxima eficiencia a la docencia de la medicina sean sustentados por la mejor evidencia disponible.

g) El impulso del profesionalismo y del humanismo en Medicina. El ejercicio de la Medicina exige, además de la actuación competente desde el punto de vista científico-técnico, actitudes y comportamientos que reflejen los valores humanos adquiridos durante la formación. Por ello, la Educación Médica debe incluir la tarea esencial de transmisión de esos valores constitutivos del profesionalismo y del humanismo médico utilizando diversas estrategias.

Todas estas consideraciones no transcienden en España y, como mucho, son conocidas por un pequeño, aunque activo grupo de profesores universitarios que, interesados por la Educación Médica, asisten regularmente a los foros internacionales donde se exponen estas ideas.

\section{La reforma curricular de 1990}

En 1986, España ingresa en la Comunidad Europea y cuatro años después, tras un largo período de discusiones ${ }^{20}$, se publica el RD1417/1990 por el que se establece el título universitario oficial de Licenciado en Medicina y las directrices generales propias de los planes de estudios, conducentes a la obtención del mismo ${ }^{21}$, de acuerdo con la Ley Orgánica de Reforma Universitaria (LRU) de $1983^{22}$ y las correspondientes Directivas de la Comunidad Europea $^{23}$. Las directrices españolas introducen los objetivos generales de aprendizaje y el concepto de crédito académico basado en horas de actividad lectiva presencial del alumno; fijan las materias troncales del plan de estudios, sus contenidos y créditos respectivos; y establecen las áreas de conocimientos encargadas de su impartición. También implantan la proporción de enseñanza teórica y práctica en cada material troncal e introducen la optatividad. Sin embargo, mantienen la tradicional separación entre el período preclínico o básico y el clínico. A pesar de evidentes limitaciones, las nuevas directrices presentan algunos aspectos positivos ya que suponen el reconocimiento del título español en los demás países 
de la Unión Europea; la posibilidad de establecer una integración horizontal y vertical de materias en cada uno de los períodos del currículum (básico y clínico respectivamente), aunque no entre ellos; la mejora del balance entre la enseñanza teórica y práctica, con un incremento muy significativo de las horas dedicadas a esta última; y la introducción de la optatividad, que debería permitir a los estudiantes diseñar, al menos en parte, su propio currículum ${ }^{1}$.

A partir de 1990 se inicia un proceso de reforma curricular en las diferentes Facultades de Medicina españolas que, de forma progresiva, van adaptando sus planes de estudio a las nuevas directivas. El proceso, sin embargo, es lento y, si bien en un principio debería haber finalizado en tres años, la realidad es que todas las Facultades de Medicina no completan la reforma hasta al menos 10 años más tarde. Lo que debería haber supuesto esta reforma y lo que en realidad ha significado ya pertenece a nuestro presente.

\section{DEL SIGLO XX AL XXI: EL PRESENTE}

¿Qué ha ocurrido en nuestras facultades desde 1994, año en que se empiezan a poner en práctica los nuevos planes de estudios, hasta los inicios del siglo XXI en que se nos plantea una nueva reforma curricular? Del análisis ${ }^{24}$ de lo ocurrido durante este período de 12 años, podemos afirmar que, en general, se ha perdido nuevamente una oportunidad para mejorar nuestra formación de grado y que se ha hecho muy poco caso de las recomendaciones de instituciones internacionales de gran prestigio, en especial de los Estándares para la Mejora de la Calidad de la WFME que antes citábamos y que marcaban claramente el camino a recorrer; recomendaciones que, por otra parte, las Facultades de Medicina españolas, entre otros agentes, habían aceptado y se habían comprometido a implementar en sus respectivas instituciones, en la denominada Declaración de Granada, $(2001)^{25}$. Analicemos con más detalle estos hechos.

\section{El modelo curricular. Contenidos y carga lectiva. Definición del tipo de licenciado a formar}

Respecto al modelo curricular pocas facultades optaron en la reforma por un modelo integrado. La integración sólo está presente en un $35 \%$ de las mismas en el período básico y en un $60 \%$ en el período clínico. En la mayoría de los planes de estudio se mantienen unos contenidos excesivos, algunos de los cua- les son irrelevantes o no pertinentes, y ello debido, fundamentalmente, al temor a perder horas lectivas y a sus posibles consecuencias sobre las dotaciones presupuestarias y de recursos de profesorado. La optatividad se contempla en muchas ocasiones como una forma de incrementar la carga lectiva utilizándola para dar aquella información que no se puede impartir en la troncalidad o para introducir contenidos nuevos que obedecen en muchos casos a intereses particulares de determinados profesores. No existe una clara definición del licenciado a formar, lo cual dificulta una planificación docente adecuada ${ }^{24}$.

\section{Las metodologías y las estrategias docentes}

Si bien los planes de estudio cambian formalmente, la metodología docente y las estrategias docentes no varían substancialmente. Se mantiene mayoritariamente una metodología docente centrada en el profesor; se potencia poco la capacidad del estudiante para identificar, formular, resolver problemas concretos y tomar decisiones mediante un planteamiento realmente multidisciplinario; y el trabajo en equipo y en pequeños grupos se estimula escasamente. Se sigue sin poner suficiente énfasis en que la evaluación es también una estrategia docente que condiciona en gran medida el éxito de un currículum y no se cambian los sistemas de evaluación sobre todo en lo que se refiere a la evaluación de las habilidades básicas y clínicas y de las actitudes ${ }^{24}$.

\section{La docencia práctica}

La docencia práctica se incrementa significativamente (50\% en el período básico y más del $60 \%$ en el clínico) y se extiende al ámbito de la atención primaria y aunque en menor medida, a los denominados laboratorios de habilidades clínicas. Sin embargo, tanto en el período básico como en el clínico, sigue faltando una clara definición de los objetivos a alcanzar en las mismas; la enseñanza práctica sigue estando poco sistematizada y con una participación poco activa del estudiante; y, finalmente, no existe una evaluación objetiva y fiable de la misma ${ }^{24}$.

\section{El control y el seguimiento interno \\ y externo de los planes de estudios}

La reforma vigente de nuestra enseñanza pregraduada vuelve a chocar contra los intereses personales o departamentales que, por regla general, siguen estando siempre por encima de los de la institución. 
Las autoridades académicas no disponen del poder necesario para imponer los intereses generales por encima de los particulares y las Facultades siguen sin recurrir al asesoramiento de expertos en Educación Médica para el desarrollo curricular, por no creerlo necesario o por considerar que ya disponen del conocimiento experto suficiente para afrontar dichas reformas. No es posible crear en las Facultades de Medicina las denominadas Unidades o Departamentos de Educación Médica, que se han convertido en habituales fuera de nuestro país y que han dado y están dando tan buenos resultados. A pesar de ello, sí que se inician los procesos de evaluación externa de la calidad de las Universidades y de las Facultades de Medicina, en los cuales se ponen de manifiesto los puntos débiles y las posibles propuestas de mejora, aunque sin el deseado el impacto en la mejora de la calidad ${ }^{24}$.

\section{El Profesorado}

Como suele ser habitual, los cambios curriculares no son bien acogidos por el profesorado, ya sea por mero desinterés por la función docente o porque las reformas exigen generalmente una mayor dedicación. Teniendo en cuenta la crónica falta de reconocimiento de la función docente en nuestro sistema universitario -en el cual las plazas docentes se siguen convocando por necesidades docentes y se resuelven, mayoritariamente, en función de méritos de investigación-, no ha de extrañar esta falta de interés por la actividad docente. Mientras ésta no se reconozca en su justa medida y al mismo nivel de la investigación, va a ser muy difícil concienciar al profesorado de que participe en reformas profundas de nuestra enseñanza de pregrado ${ }^{24}$.

\section{Los estudiantes}

¿Cómo han acogido nuestros estudiantes la reforma? En general, reconocen que las cosas han mejorado en determinados aspectos, pero siguen siendo críticos con la misma y en general con cualquier tipo de cambio que en su opinión les suponga un esfuerzo excesivo y no les sea útil para su fin último, que sigue siendo el de alcanzar un buen resultado en el examen de acceso a la formación especializada, examen que sigue primando el componente de conocimientos sobre otros ${ }^{24}$.

En resumen, la reforma de 1990, basada en unas directrices algo restrictivas en ciertos aspectos, supone cierta mejoría respecto a la situación anterior que databa de 1974. Sin embargo, no es aprovechada para introducir cambios substanciales en nuestros curricula. Dieciséis años después, se mantienen en la enseñanza de grado diversos problemas, algunos de los cuales se vienen arrastrando desde hace más tiempo y que podemos sintetizar en los siguientes puntos ${ }^{24}$ :

1. La función docente sigue estando poco valorada en comparación con la investigadora.

2. Se siguen impartiendo excesivos contenidos teóricos y frecuentemente irrelevantes o de escasa utilidad para nuestros estudiantes en el momento de iniciar su práctica profesional, sobre todo si se considera la velocidad con que se generan nuevos conocimientos científicos. La enseñanza impartida sigue estando orientada básicamente a proporcionar información y continúa centrada en el profesor, en vez de estarlo en el aprendizaje del estudiante y en que éste sea capaz de adquirir el hábito del aprendizaje autónomo.

3 . Se sigue empleando una metodología poco activa y basada en la lección magistral.

4. La docencia práctica, si bien se ha incrementado en cantidad, no ha mejorado excesivamente en su calidad. Las actividades prácticas, tanto básicas como clínicas, adolecen en muchos casos de falta de pertinencia o de exceso de pasividad por parte del estudiante.

5. Las metodologías de evaluación que se utilizan están centradas fundamentalmente en la evaluación de los conocimientos teóricos.

6. Sigue persistiendo una frontera infranqueable entre el período preclínico y clínico que impide una integración real básica-clínica

7. Sigue existiendo, a la hora de gestionar la docencia del grado, una excesiva autonomía de los departamentos.

8. No se han creado Unidades de Educación Médica, a través de las cuales expertos en Educación Médica puedan brindar a los docentes la ayuda necesaria en temas educativos.

9. Existe una falta evidente de recursos humanos cualificados y de recursos materiales para introducir cambios reales.

10. Los actuales planes de estudio siguen sin tener en cuenta los valores del profesionalismo.

\section{LOS RETOS DEL SIGLO XXI: EL FUTURO}

En 1999 se inicia, con la denominada Declaración de Bolonia ${ }^{26}$, la construcción del EEES. Este proceso pretende, fundamentalmente, adoptar un sistema de 
titulaciones comprensibles y comparables (mediante un sistema de titulaciones basado en dos ciclos); establecer un sistema común de créditos (ECTS, European Credit Transfer System), basado en el trabajo del estudiante; la formación basada en competencias; el fomento de la movilidad y de la cooperación europea para garantizar la calidad; estimular el aprendizaje a lo largo de la vida; la promoción de una dimensión europea en educación superior; promover el atractivo del Área Europea de Educación Superior; y el establecimiento de estrechas relaciones entre los espacios europeos de Educación Superior y de Investigación.

Por lo que se refiere a los estudios de Medicina, el proceso se ha vivido en general como algo relativamente alejado, poco concerniente y con bastantes reservas, entre otras razones porque los estudios de Medicina disponen de una Directiva Europea propia. A nivel europeo, es a partir del año 2004 cuando el tema empieza a ser motivo de discusión en diferentes foros o reuniones [Congresos de la Association for Medical Education in Europe (AMEE), 2004, 2005; Ottawa Conference 2004, Reuniones de la IMFSA, 2004, 2005, reuniones del Comité Permanent des Médecins Européens, 2004, reuniones de la WFME, y reuniones de la red MEDINE o Thematic Network on Medical Education in Europe] ${ }^{27-32}$. Sin duda alguna, las dos aportaciones más importantes a la cuestión son la declaración conjunta de la AMEE y la WFME y los trabajos de la red MEDINE. En la primera $^{33}$ se respalda la Declaración de Bolonia y se insiste en la necesidad de que la Educación Médica, como parte de la educación superior, se implique totalmente en el proceso de convergencia europea. Se afirma que la mayoría de los objetivos de Bolonia están de acuerdo con las prácticas actuales de la Educación Médica y con las reformas emprendidas por las Facultades de Medicina; si bien se hacen ciertas matizaciones sobre algunos de los objetivos, que se considera deben desarrollarse con miras más amplias, como el establecimiento de titulaciones comprensibles y comparables, la aplicación del sistema ECTS, la promoción de la movilidad, el desarrollo de garantías de calidad, la formación continuada, y la implicación de las instituciones y de los estudiantes en el proceso. Pero también se alerta sobre un objetivo cuya implementación podría tener serias consecuencias para la calidad de la Educación Médica y que ya había sido denunciado en los pronunciamientos de la IFMSA/EMSA y de la
CPME: la estructuración en dos ciclos (de grado y de postgrado) de los estudios universitarios. En la Declaración conjunta de la AMEE y la WFME se analizaron las razones existentes para oponerse y se propuso que las Facultades de Medicina no se vieran obligadas a adoptar dicha estructura, de forma que se les permitiera mantener los estudios estructurados en una unidad integrada más prolongada. Se afirmaba que, alternativamente, se les debería dar la opción de establecer un primer ciclo como primera parte del programa formativo, pero sin tener que planificarlo con el fin de preparar para actividades vocacionales o profesionales especiales. El documento finalizaba urgiendo a los gobiernos para que tomasen aquellas decisiones que serán críticas para la Educación Médica en base a la necesaria evidencia y previo diálogo con las Facultades de Medicina y sus agentes. Por su parte la Red MEDINE ${ }^{32}$ persigue cinco objetivos fundamentales: 1) acordar las competencias/objetivos formativos nucleares de la Educación Médica en Europa utilizando la metodología del Proyecto "Tuning Educational Structures in Europe"; 2) desarrollar una estructura para el reconocimiento internacional de las calificaciones en el ámbito de la formación médica y en conexión con otras profesiones; 3 ) desarrollar estándares de garantía de calidad en el proceso de formación médica aplicables en Europa; 4) incrementar la transparencia y el conocimiento por parte de la sociedad en general del proceso de formación médica (de grado, postgrado y continuada) y de sus objetivos de aprendizaje, dentro y fuera de Europa; 5) explorar y desarrollar conexiones entre proceso formativo e investigación.

¿Qué ocurre en España en este tiempo? A partir del año 2005 en que se publican los decretos que regulan los estudios oficiales universitarios de Grado y Postgrado34, se empieza a producir cierta movilización y el tema empieza a ser objeto de discusión en talleres, seminarios, jornadas y cursos organizados por las Facultades, por las universidades y por las Agencias de Evaluación de la Calidad. Diversos aspectos del proceso de convergencia europea y sus repercusiones en las Facultades de Medicina, también han sido abordados por distintas sociedades de Educación Médica de nuestro país y, muy especialmente, en el XVII Congreso de la SEDEM celebrado en Albacete en octubre de $2005^{35-38}$. Por otra parte, se han desarrollado dos acciones de especial importancia: una de ámbito estatal, la elaboración por la 
Conferencia Nacional de Decanos de Facultades de Medicina Españolas del "Libro Blanco de la Titulación de Medicina” ${ }^{39}$, y otra de ámbito catalán, la publicación del documento "Competencias profesionales básicas comunes de los licenciados en Medicina formados en las universidades de Cataluña"40.

El aspecto más controvertido hasta la fecha ha sido el del nivel académico correspondiente a los estudios de Medicina, relacionado directamente con lo propuesto en la declaración de Bolonia de establecer un sistema de titulaciones basado en dos ciclos. De hecho la indefinición en este punto ha retrasado de forma evidente la incorporación de las Facultades de Medicina a este proceso, por no saber exactamente a qué atenerse. Finalmente, en abril de 2006, y con el fin de adaptarse al EEES, el Ministerio de Educación y Ciencia ha hecho público el borrador de la Ficha Técnica de Propuesta de Título Universitario de las Enseñanzas de Medicina ${ }^{41}$, y que ha constituir la base de las directrices de elaboración de los nuevos planes de estudio de Medicina en España. La ficha, entre otros aspectos que analizaremos posteriormente, establece que los estudios de Medicina constituyen unos estudios de Grado de seis años, de ciclo único, sin divisiones entre período básico y clínico, como venía siendo tradicional, y permite a los futuros licenciados acceder directamente a los estudios de Doctorado. Además, la ficha contiene otros aspectos positivos como $^{42}$ :

La gran flexibilidad que otorga a las Facultades de Medicina para la elaboración de sus respectivos planes de estudios.

El carácter continuo que otorga al proceso formativo, no diferenciando entre ciclo básico y clínico.

Las recomendaciones en relación a la necesidad de integrar los contenidos tanto de forma horizontal como vertical; a que el estudiante entre precozmente en contacto con las disciplinas clínicas; a la presencia de las materias básicas en los cursos finales; al establecimiento de mecanismos globales de evaluación de las competencias; y al hecho de tener en cuenta en la estructuración de los planes de estudio y en el establecimiento de los programas, la necesaria continuidad con la formación especializada.

La distribución en créditos y la adecuación al EEES de las diferentes grandes materias consideradas.

Despejada la incógnita del nivel académico de los estudios de Medicina, que tanto preocupaba, y fijada la ficha técnica -hemos de suponer que la definitiva no variará en demasía respecto al borrador-, las Facultades de Medicina Españolas ya pueden abordar este nuevo proceso de reforma curricular que se ofrece como una oportunidad única para adaptar nuestros estudios de Medicina a las tendencias más modernas en Educación Médica y que ya están vigentes en muchas Facultades de Medicina europeas. Conscientes de nuestros defectos y ante este nuevo proceso, no podemos caer en la tentación, como ha ocurrido en otras ocasiones, de llevar a cabo una reforma superficial o un mero maquillaje de lo que tenemos hoy en día. Debemos ser conscientes de que nuestro principal reto es acometer una reforma real y en profundidad de nuestra enseñanza graduada que nos haga converger con Europa y en particular con aquellos países en los que se ha llevado a cabo un cambio real con resultados positivos contrastados. No se trata de inventar nada. Otros antes que nosotros han acometido reformas reales e innovadoras que se han demostrado útiles y positivas. La literatura en Educación Medica en este sentido es abundante y a ella nos podemos remitir. Incluso en nuestro país, una Facultad nueva, creada en 1998, la de la Universidad de Castilla-La Mancha, en Albacete, ha puesto en marcha un modelo innovador con resultados que parecen ser positivos ${ }^{43}$.

De entre los documentos que más útiles van a ser para guiarnos en nuestro proceso de reforma, destacan los ya previamente citados Estándares de Calidad para la Educación Médica graduada de la WFME $(2001)^{18}$ y el elaborado por el Instituto de Educación Médica Internacional (IIME) ${ }^{44}$, que define las competencias generales que ha de demostrar cualquier médico independientemente de donde se forme. Con respecto al primero debemos recordar nuevamente que dicho documento fue asumido en 2001, en la denominada Declaración de Granada, por la Conferencia Nacional de Decanos de Facultades de Medicina, la Organización Medica Colegial, los Ministerios de Educación y Sanidad y diversas Sociedades Científicas como la SEDEM, la Associació Catalana d'Educació Mèdica (ACEM) y la Sociedad de Educación Médica de Euskadi (SEMDE). Por su parte, la Declaración de Granada ha sido tenida en cuenta como referente por la Conferencia de Decanos para la elaboración del Libro Blanco.

¿En qué se traduce este reto? En primer lugar, como dice $\mathrm{Oriol}^{45}$, hemos de considerar que un currículum o un plan de estudios, no es únicamente la 
norma publicada en el BOE y que sólo describe lo que es imperativo que las Facultades de Medicina hagan en relación con la formación de sus estudiantes, junto con el listado de materias, asignaturas y cargas lectivas que corresponde a cada una de ellas, así como su ordenación por períodos lectivos. El concepto de currículum es algo más complejo que la simple norma. Actualmente, el término "currículum" incluye las estrategias educativas utilizadas, los contenidos y los objetivos de aprendizaje, las experiencias educativas, el entorno educativo, la evaluación, los estilos y ritmos de aprendizaje, la programación de tareas y el programa y resultados del aprendizaje. Por tanto, a la hora de enfrentarnos a este reto, hemos de tener en cuenta todos estos aspectos.

\section{El modelo de médico y sus competencias:}

En primer lugar deberíamos aceptar que, en nuestro contexto, el modelo de médico a formar es aquel que al final de sus estudios de grado posea todas aquellas competencias básicas y comunes a todos ellos/ellas, (entendiendo las competencias como combinaciones dinámicas de conocimientos, habilidades, actitudes y valores) que les permita, en un primer momento, ejercer una Medicina tutelada en nuestro sistema sanitario y acceder a la formación especializada en cualquiera de las disciplinas, así como iniciarse en la investigación biomédica y, eventualmente, en la docencia. Aceptado este modelo de médico, el siguiente paso es establecer correctamente sus competencias profesionales de forma detallada. Las Facultades de Medicina (algunas ya lo han hech ${ }^{46,47}$ ), deben iniciar un proceso de definición de las competencias que sus estudiantes han de haber adquirido al final de su formación de grado de acuerdo con el modelo determinado. Y deben aceptar la participación en el proceso de otros agentes que están claramente involucrados en dicha formación, como son las Administraciones Educativas y Sanitarias, el Sistema Publico de Salud, la Organizaciones Profesionales, otros profesionales de las Ciencias de la Salud, los estudiantes, los graduados y, de forma muy especial, los tutores de postgrado que van a recibir en última instancia a los graduados formados en las Facultades. Las Facultades de Medicina no pueden vivir de espaldas a la formación postgraduada o especializada y las competencias definidas han de estar perfectamente interrelacionadas con la formación postgraduada. Hemos de ser capaces de definir las competencias a dos niveles: competencias nucleares mínimas y comunes a todos los médicos sea cual sea la Facultad de Medicina donde se hayan formado, y aquéllas otras competencias más específicas que las Facultades consideren necesarias para sus propios estudiantes. En este sentido, no debe darnos miedo introducir en los planes de estudio un determinado grado de variabilidad entre las Facultades que sin duda enriquece la formación de nuestros graduados. En este proceso de definición de competencias, no debemos inventar nada. Podemos recurrir a los múltiples modelos que otras facultades fuera de nuestro país han utiliza$\mathrm{do}^{44,48}$.

\section{El modelo, las estrategias curriculares y las metodologías docentes}

Bolonia nos pide que formemos a nuestros graduados en competencias profesionales. Es decir, nos está diciendo que definamos planes de estudios orientados a las competencias finale ${ }^{49}$, y ello exige que el modelo, las estrategias curriculares y las metodologías que adoptemos nos permitan alcanzar este objetivo. Para ello debemos aceptar que:

1. El modelo curricular ha de poner mayor énfasis en el desarrollo de las competencias, que en la mera transmisión de información y de conocimientos teóricos. Hemos de ser capaces de reducir en todo lo posible el exceso y la redundancia o irrelevancia de la información. Para ello es necesario llevar a cabo una revisión en profundidad de los contenidos actuales por encima de los intereses personales y/o departamentales.

2. Un currículum basado en competencias es, por definición, un currículum integrado. La literatura en Educación Médica nos dice hasta la saciedad que un currículum integrado, en cualquiera de sus modalidades, posee evidentes ventajas sobre uno tradicional basado en disciplinas, facilita el contacto precoz del estudiante con la realidad médica, les permite captar mejor la relevancia de las disciplinas básicas ${ }^{50,51}$ y favorece el trabajo en equipo y multidisciplinar. Hemos de ser capaces de introducir este modelo de currículum aún a pesar de aquellas voces que nos dicen que los curricula integrados son inviables. Por otra parte, la ficha técnica publicada por el Ministerio de Educación y Ciencia permite, al haber anulado la tradicional división entre dos ciclos básico y clínico, el establecimiento de este tipo de currículum.

3. Los modelos curriculares y los métodos educati- 
vos seleccionados deben asegurar una preparación real de los estudiantes para que se responsabilicen de su propio proceso de aprendizaje y para un proceso de aprendizaje autónomo de por vida. Por ello debemos intentar adoptar aquellas metodologías de tipo activo, huyendo en lo posible de la clase tradicional todavía tan presente en nuestro entorno. Estrategias como el aprendizaje basado en problemas (ABP), entre otras, son sin duda opciones a tener en cuenta. Si bien el ABP es considerado como un paradigma, más que como una metodología docente, y existe una tendencia a pensar que se requiere que todo el currículum esté estructurado a su alrededor, debido a que las experiencias que conocemos provienen de este tipo de contexto, nada impide que en determinadas materias o unidades podamos programar la enseñanza en forma de $\mathrm{ABP}$ y no podemos renunciar a ello ${ }^{52-54}$.

4. La ficha técnica fija únicamente los créditos de cada una de las materias del currículum. La distribución interna corresponde a cada Facultad. Pero, en cualquier caso, no se debe empezar a construir la casa por el tejado. Esto es, no se deben distribuir los créditos antes de fijar de forma clara cómo contribuye cada área de conocimiento a la adquisición de cada una de las competencias y mediante qué tipo de actividades docentes lo hace. Sólo después de concretar esto podremos establecer los créditos para cada unidad, módulo o asignatura.

\section{Los entornos educativos}

Para asegurar una correcta adquisición de las competencias de nuestros estudiantes hemos de ser capaces de formarlos en todos los entornos educativos posibles. Si bien el entorno hospitalario ha de ser tenido en cuenta, éste ya no es, ni puede ser, el único. Se ha de utilizar, inexcusablemente, el entorno de la atención primaria, en el cual un elevado porcentaje de nuestros estudiantes ejercerán su profesión. El énfasis puesto, por un lado, en la necesidad de que los estudiantes adquieran las habilidades básicas, la capacidad de razonamiento clínico y la necesidad de fomentar su aprendizaje autónomo; y, por el otro, los cambios en el modelo asistencial que imposibilitan que un paciente pueda ser sometido de forma repetida a exploraciones y procedimientos con objeto de entrenar a los estudiantes, hace ineludible adecuar nuevos entornos educativos para la formación de nuestros alumnos en habilidades clínicas. El impre- sionante desarrollo en el campo de las simulaciones permite, y podríamos decir que obliga a las Facultades de Medicina a plantearse seriamente el reto de disponer de los denominados laboratorios de habilidades clínicas, entendidos como entornos educativos perfectamente integrados en el currículum y en la actividad docente de la Facultad. En ellos los estudiantes y los profesionales médicos o sanitarios pueden aprender diferentes tipos de habilidades en un entorno no estresante, bajo la supervisión de profesores o de forma autónoma e independiente. Además, es posible repetir, tantas veces como se desee, la técnica o habilidad concreta; el error está permitido, el estudiante recibe de forma continua feed-back sobre su actuación, y puede ser evaluado de forma objetiva ${ }^{55-58}$. Como dice Amitai Ziv, Director del Israel National Simulation Center, Chaim Sheva Medical Center, "la educación médica basada en simulaciones es hoy día un imperativo ético. Asegurar la seguridad de los pacientes entra en conflicto con el aprendizaje de los futuros profesionales en algún momento del proceso educativo. Las simulaciones pueden minimizar este conflicto ético" ${ }^{\text {59. }}$.

También debemos replantearnos el concepto de lo que conocemos como prácticas básicas. Los objetivos de las mismas deben estar perfectamente incardinados y ser relevantes para la adquisición de las competencias finales establecidas por la Facultad.

\section{La evaluación}

La evaluación es el elemento que más condiciona el proceso de aprendizaje. Un currículum basado en competencias exige evaluar dichas competencias. La evaluación de las competencias debe utilizar instrumentos válidos, fiables y factibles. El diseño que se adopte debe tener en cuenta su aceptabilidad e impacto educativo tanto para los estudiantes como para los profesores. Todos los agentes implicados, tanto docentes como discentes, deben participar activamente en el proceso. En este sentido, se plantea un nuevo reto: la incorporación de los docentes en el proceso de evaluación y su formación en los distintos métodos existentes, alguno de los cuales, y en especial las ECOES (evaluación clínica objetiva estructurada $)^{60,61}$, ya se vienen utilizando de forma general fuera de nuestro país y de forma más esporádica en algunas de nuestras Facultades. Sería deseable que las Facultades afrontaran cuanto antes el reto de adquirir experiencia en la evaluación de las compe- 
tencias, para lo que dicho proceso debe iniciarse lo antes posible e implantarse en todo el currículum.

Con independencia de la estrategia de implantación continuada de aprendizaje y de evaluación de competencias a lo largo de toda la carrera, y como garantía ante la sociedad de la adquisición de las competencias, las Facultades deberían plantearse muy seriamente establecer al final de los estudios de grado una evaluación formal, dotada de validez académica, de las competencias adquiridas. Conjuntamente con otros elementos, esta evaluación final de las competencias debería ser reconocida tanto por las asociaciones profesionales como por los programas de formación postgraduada, lo que permitiría, al menos en parte, aliviar la hipoteca que ejerce el examen MIR sobre la enseñanza pregraduada. Pero ello no es suficiente. Sin duda alguna uno de los grandes retos que tiene la enseñanza de grado en nuestro país es la reforma del tipo de examen que da acceso a la formación especializada. Sin dicha modificación, la enseñanza de grado seguirá estando muy condicionada. ${ }^{10,37}$

\section{El profesorado}

Una reforma comprometida y responsable requiere recursos humanos adecuados. Sin duda, al menos desde un punto de vista cuantitativo, disponemos de estos recursos. La ratio alumno/profesor actual de las Facultades de Medicina no es malo ${ }^{62}$. Pero una reforma como la que se inicia requiere una formación adecuada y continua del docente que, hoy en día, de forma general, no posee. Nuestro reto es que el profesor aprecie la necesidad de esta formación y se implique en ella y en la reforma docente. Para ello es necesario el reconocimiento, de una vez por todas, de la función docente, y el establecimiento de criterios objetivos para su evaluación, asumidos tanto por los interesados como por las autoridades académicas. ${ }^{37}$

\section{Los estudiantes}

Los estudiantes son los usuarios de nuestra docencia. Por lo general son reacios a participar en reformas, sobre todo en aquellas que preconizan un mayor trabajo individual y autónomo. Es preciso hacerles ver la necesidad de que sean corresponsables de su formación, y que es fundamental que durante los estudios de grado, además de conocimientos, habilidades y actitudes, aprendan a aprender durante toda su vida profesional. Las
Facultades, asimismo, han de poner en marcha todos los mecanismos que aseguren esta formación.

\section{Los valores del profesionalismo en la formación médica pregraduada}

Es universalmente aceptado que el ejercicio de la Medicina es un cometido moral. Es una profesión cuyos miembros comulgan con una serie de principios válidos desde hace mucho tiempo, como el respeto hacia los demás, la empatía, la compasión, la honestidad, la integridad, el altruismo y la excelencia profesional. El concepto de Profesionalismo Médico encuentra sus raíces en estos principios. En la actualidad, estos principios chocan con la transformación corporativa que se está produciendo en la asistencia médica, que tiende de forma creciente hacia un sistema de salud cada vez más imbuido por aspectos economicistas y donde las claves son el coste, los beneficios y la competitividad ${ }^{63}$.

Desde 1988, fecha de la Declaración de Edimburgo, hasta nuestros días, diferentes instituciones han venido insistiendo en la necesidad de formar a nuestros estudiantes, los médicos del futuro, en los valores del profesionalismo. Entre estas instituciones destaca la posición del General Medical Council (GMC) británico, que en su ya citado documento "Tomorow's Doctors"16,17, recomienda que los estudiantes de grado se formen "en el respeto por los pacientes y colegas, lo que incluye no mostrar prejuicios frente a la diversidad de orígenes y oportunidades, idiomas, cultura y formas de vida... en el reconocimiento de los derechos de los pacientes en todos los aspectos y particularmente la confidencialidad... y en tomar conciencia de las responsabilidades éticas y morales de la asistencia a los pacientes individuales $y$...de las poblaciones...y de las propias limitaciones..." etc. El GMC publicaba asimismo el documento Good Medical Practice ${ }^{64}$ en el que aconsejan pautas de honradez en la información sobre los servicios prestados, en los informes, en los tratos comerciales y financieros, en la investigación o en los conflictos de intereses. Por su parte, los Requerimientos Globales Esenciales Mínimos elaborados por el Institute for Internacional Medical Education $^{44}$ señalaba la necesidad de formar a los estudiantes en los valores profesionales y éticos que subyacen a las actitudes y conforman las conductas.

En nuestro entorno algunas Facultades de Medicina que han definido sus competencias hacen mención de los valores profesionales y el profesiona- 
lismo, sin menoscabo, como es natural, de las capacidades científico-técnicas y clínicas que son exigibles $^{46,47}$.

De acuerdo con Wojtczak ${ }^{63}$, se debe concienciar a los médicos, durante su período formativo, de la existencia de los conflictos, antes indicados, entre las obligaciones tradicionales y los cambios existentes en los sistemas sanitarios, de forma que estén mejor preparados para defender sus valores profesionales en este nuevo clima economicista. Jordan Cohen, exPresidente de la Asociación de Colegios Médicos Americanos, en su alocución en la Convención Anual de esta asociación, insistía en que "la actual obligación de la profesión médica es cultivar los valores nucleares del profesionalismo en los futuros médicos $y$ mantenerse firmes en el soporte de estos valores que hacen de nuestra profesión una profesión honrada y honorable". ${ }^{63}$

Los médicos que actuarán en la primera mitad del siglo XXI están ya en formación en nuestras Facultades de Medicina. Lo que estén haciendo y puedan hacer los sistemas educativos definirá el tipo de médico del que dispondremos en los sistemas asistenciales $^{65}$. Por ello nuestras Facultades han de ser muy conscientes de la necesidad ineludible de formar a sus estudiantes en los principios que definen el profesionalismo médico y adoptar aquellas medidas y estrategias docentes en sus respectivos curricula, que lo hagan posible.

\section{Los recursos materiales}

Una reforma comprometida y responsable requiere también la aplicación de los recursos materiales necesarios. Estos recursos se han de aplicar a disponer de las infraestructuras precisas (laboratorios básicos, laboratorio de habilidades y recursos sanitarios suficientes). En el futuro inmediato, estos recursos serán cada vez mayores ya que se advierte una tendencia a incrementar progresivamente el número de alumnos que ingresan en las Facultades de Medicina. Si la anterior reforma se hizo en muchos casos a coste cero, no puede suceder lo mismo en la actual. Afrontar el reto de una reforma en profundidad supone que las Administraciones Educativas y Sanitarias a todos los niveles colaboren estrechamente entre sí, siendo activas en la búsqueda y facilitación de dichos recursos.

\section{La gestión del programa educativo}

La elaboración de un currículum y su proceso de implantación es un proceso complejo, largo y difícil. Por otra parte un currículum no es algo inmutable y, por tanto, mientras permanezca vigente debe estar sometido a continua evaluación y revisión, debiéndose introducir los cambios que sean pertinentes siempre que sea necesario, ya que tanto en Educación Médica como en la práctica médica los cambios son constantes.

Para que todo este proceso se desarrolle correctamente, es preciso -tal como indican claramente los Estándares de Calidad para la formación médica básica de la WFME- que exista en el seno de las Facultades de Medicina un comité curricular o de programa independiente que incluya profesores, estudiantes y otros agentes. Este comité ha de tener toda la responsabilidad y, sobre todo, toda la autoridad para programar y implementar el currículum por encima de intereses departamentales o individuales. Los profesores han de formar parte, conjuntamente con la autoridad académica, de este comité curricular. Hemos de ser conscientes, sin embargo, de que los profesores, por regla general, no suelen ser expertos en temas de Educación Médica. Ni es necesario que todos lo sean. Pero sí deben ser conocedores de lo que está pasando o ha pasado en el mundo educativo, $\mathrm{y}$ ser conscientes de que se puede mejorar haciendo las cosas de manera diferente, en función de lo que han experimentado otros para obtener mejoras reconocidas. Por ello es necesario recurrir a la experteza en el campo de la Educación Médica. Los Estándares de Calidad de la WFME proponen que todo el proceso de diseño y gestión de un proceso curricular se profesionalice mediante la participación de expertos en Educación Médica, que asesorarán a los diferentes comités curriculares. Si bien en un primer momento esta experteza educativa puede y debe obtenerse de una institución nacional o internacional externa, lo óptimo sería que todas las Facultades de Medicina crearan, a medio plazo, unidades o Departamentos de Educación Medica, de los cuales tenemos en España actualmente alguna experiencia (Facultad de Medicina de la Universidad de Castilla-La Mancha, Albacete $)^{45}$. En el último congreso de la SEDEM, celebrado en Albacete, hubo un consenso generalizado sobre la conveniencia de disponer de este tipo de unidades. Esperemos que tras este consenso, las Facultades de Medicina afronten la necesidad de estas estructuras y se decidan, de una vez por todas, 
a recurrir a un conocimiento experto en Educación Médica, para, posteriormente, crear este conocimiento experto y no continuar como hasta ahora pensando que ya lo tienen. ${ }^{20,40}$

\section{Evaluación y acreditación de las Facultades de Medicina}

Por último, aunque no menos importante, otro reto fundamental al que nos tendremos que enfrentar en un futuro próximo será el relacionado con los procesos de evaluación y acreditación de las Facultades de Medicina y de sus programas de formación. Uno de los objetivos del proceso de Bolonia es la garantía de la calidad de las instituciones y de sus programas de formación. La mejora de la calidad y la excelencia del proceso enseñanza/aprendizaje son los objetivos que deben presidir los programas para la acreditación de las instituciones docentes y, por tanto, su aplicación práctica. La evaluación de las instituciones docentes públicas persigue además rendir cuentas ante la sociedad sobre el grado de cumplimiento de su misión y sobre su eficacia de la gestión de fondos públicos. La calidad de la docencia viene determinada por la estructura y el proceso, pero también por la evaluación competencial de los egresados y su adecuación a las demandas sociales. En el área de la Medicina es de particular importancia contemplar las necesidades sociales y futuras de Salud, la evolución del proceso científico y tecnológico, las variaciones demográficas que puedan tener lugar y otros cambios para ajustar la docencia a estas previsiones y, consecuentemente, adaptar los esquemas de evaluación de las mismas ${ }^{66}$. Los procesos de evaluación y acreditación han de realizarse confrontando la realidad de cada institución con estándares claramente definidos. En este sentido debemos citar nuevamente los Estándares Globales en Educación Médica para una mejora de la Atención de Salud, publicados por la $\mathrm{WFME}^{20}$. Así mismo, esta organización, conjuntamente con la OMS, y con el fin de facilitar la labor evaluadora y acreditadora de cada uno de las agencias nacionales o regionales, ha elaborado una guía para la acreditación de la formación médica de grado ${ }^{67}$. Esta guía ha sido desarrollada como un instrumento de ayuda para las autoridades y agencias nacionales responsables de la calidad de la Educación Médica, con el fin de garantizar el funcionamiento y la transparencia adecuada de los sistemas de acreditación exis- tentes, o bien como un instrumento que sirva de ayuda para la puesta en marcha, en aquellos países o regiones en los que hasta ahora no existían, nuevos sistemas de acreditación. En los últimos años, en España se han llevado a cabo procesos de evaluación de las instituciones docentes por agencias nacionales (ANECA) o autonómicas, pero el impacto de los mismos sobre la calidad y excelencia del proceso ha sido más bien escaso, entre otras razones porque no ha ido acompañado de la puesta en marcha de Programas para la Mejora y la Garantía de Calidad, basados en el diagnóstico obtenido. Es totalmente necesario generalizar el proceso y por ello las Facultades de Medicina han de afrontar sin miedo el reto de ser evaluadas, ellas mismas y sus programas docentes, con el objetivo final de poder arbitrar propuestas de mejora de la calidad.

\section{Conclusiones}

Partiendo de una revisión del pasado reciente, hemos analizado los diferentes retos a que se enfrenta, en los albores de una nueva reforma -que esperamos que esta vez sea real-, la enseñanza en nuestras Facultades de Medicina. La reforma es necesaria, posible e, incluso, obligada. Experiencias exitosas en nuestro propio país nos lo demuestran. Por primera vez, las directrices emanadas de la Administración Educativa son suficientemente flexibles y permiten la reforma, como nunca antes lo habían hecho. Las Facultades de Medicina han de aprovechar esta nueva oportunidad, que es posible que tarde en presentarse otra vez. Es evidente que no todas las Facultades de Medicina son iguales. Por sus propias peculiaridades, a algunas les será más fácil que a otras avanzar en el camino de la reforma. También es verdad que hay factores que no dependen de las Facultades en sí mismas, pero sin duda, las Facultades han de ser el motor que impulse dichas reformas. Y cuando se habla de las Facultades, se quiere decir sus profesores y sus alumnos. Seamos generosos, profesores y estudiantes. Dejemos de pensar en nuestros intereses personales y departamentales y hagámoslo en los de las Facultades y en aquello que conviene realmente para impartir una docencia de calidad que permita homologarnos con los países europeos más avanzados en la mejora de la Educación Médica. Esta es la verdadera convergencia a la que debemos aspirar y este es nuestro gran reto.

\section{Bibliografía}


1. Barón, M. Innovation in Spanish Medical Education. Hopes and frustrations. In MedEd-21: An Account of Initiatives for Change in Medical Education in Europe for the 21st Century. Eds. G.D. Majoor, CPM. Van der Vleuten, PMJ. Vluggen and PA. Hansen, pp 75-85, Thesis Publishers, Amsterdam, 1997.

2. Danon, J. L'ensenyament de la Medicina a la Universitat Espanyola. Col-lecció històrica de Ciències de la Salut, vol. 6 . Fundació Uriach, Barcelona. 1998.

3. Gallego, A. Medical Studies in Spain. Journal of Medical Education, 37, 892-905, 1962.

4. Gallego, A. Medical Education in Spain. Journal of Medical Education, 46,b658-669, 1966.

5. Gallego, A. Y Botella Llusiá, J. Comentarios al nuevo plan de estudios. ARch. Fac. Med. Madrid. 4:187, 1967.

6. Belmonte, C. El modelo docente de Alicante. JANO 40, 62, 1991.

7. Resolución de la Dirección General de Ordenación Universitaria de 27 de septiembre de 1972 (BOE de 4 de octubre), sobre directrices de planes de estudio de Facultades de Medicina, complementada por otra de 19 de diciembre de 1972 (BOE de 5 de enero de 1973).

8. Real Decreto 127/1984, en el que se regula la formación especializada y la obtención del título de médico especialista, BOE $\mathrm{n}^{\circ} 26$, de 31-1-1984.

9. Segovia de Arana JM, Pera Blanco-Morales C, Goirena de Gandarias JJ, Cabasés Hita JM. La formación de los profesionales de la salud. Bilbao: Fundación BBV, Informe 1999

10. Rozman, C. Reflexiones sobre la universidad en el ámbito biomédico. Educación Médica, 8(4): 183-190, 2005.

11. World Federation for Medical Education. World Conference on Medical Education. Report, Edinburgh 7-12 August, 1988.

12. O.M.S. Ministerial Consultation for medical education in Europe. The Lisboa Initiative. 1998.

13. Walton, HJ. Proceedings of the World Summit on Medical Education . Medical Education 28 (supp 1), 140-149, 1993

14. Boelen, C. The 5-Star Doctor. MedEd in Europe for the 21st century. University of Limburg. Vaals: Maastricth Faculty of Medicine, 1995

15. O.M.S. Médecins pour la santé. Une stratégie mondiale de l'OMS pour la réorientation de l'enseignement de la médecine et de la pratique médicale en faveur de la santé pour tous. WHO/HRH/96.1. 1996.

16. General Medical Council. Tomorrow's Doctors. Recommendations on undergraduate medical education, 1993

17. General Medical Council Tomorrow's Doctor : Recommendations on Undergraduate Medical Education. (www.gmc.uk/ orgmed_ed/tomdoc.htm) 2002

18. World Federation for Medical Education: Estándares Globales en Educación Médica. Educación Médica vol 7, Supl. 2, JulioSeptiembre 2004

19. Barón, M. La formación médica en Canadá como modelo de intervención profesional. Educación Médica, 4 n² 2, 52-65, 2001.
20. Consejo de Universidades. Informe técnico del Grupo de Trabajo $\mathrm{n}^{\circ}$ 9. Título de licenciado en Medicina y Cirugía. Madrid, 9 de abril de 1987.

21. Directrices generales propias de los planes de estudios conducentes a la obtención del título oficial de Licenciado en Medicina. RD 1417/1990, de 26-10. BOE n 278, pp. 34344-34445.

22. Ley Orgánica de Reforma Universitaria, (Ley Orgánica 11/1983 de 25 de agosto, de Reforma Universitaria, B.O.E. del 1 de septiembre).

23. Directiva comunitaria $75 / 363$

24. Palés, J. Análisis del proceso de implantación de los nuevos planes de estudio de la Licenciatura de Medicina. Educación Médica, Vol 2, $\mathrm{n}^{\circ} 3$ 102-104, 2000.

25. Declaración de Granada, Educación Médica, 5, 1, pp. 9-11, 2002

26. Bologna Declaration. The European Higher Education Area. Joint Declaration of the European Ministers of Education (Bologna), 1999.

http://www.crue.org/decblognaingles.htm

27. Christensen L. The Bologna Process and Medical Education. Med Teacher 26:625-629, 2004.

28. AMEE Annual Meeting Edinburgh. Symposium. The Bologna Declaration and its implications for Medical Education. AMEE 2004 , Abstracts: 4.30.

29. AMEE Annual Meeting Amsterdam. Symposium. Bologna process and Medical Education. AMEE 2005, Abstracts: 88.

30. 11th International Ottawa Conference on Medical Education. Parallel session: Bologna Agreement. Scientific Program: 66.Barcelona 2004.

31. IFMSA. The Bologna Declaration and Medical Education: A Policy Statement from the Medical Students of Europe, Med Teacher, 27: 83-85, 2005.

32. University of Bristol. MEDINE. Medical Education in Europe. 2005.http://www.bri.ac.uk/medine/

33. WFME and AMEE. Statement on the Bologna Process and Medical Education.

http://www.amee.org/Bologna\%20final\%20Mar $\% 2005$.doc. 2005

34. Real Decreto 55/2005, 21 de enero por el que se establece la estructura de las enseñanzas universitarias y se regulan los estudios universitarios de Grado. BOE, n 21: 2842-2846, 2005.

35. XVI Congreso Nacional de la Sociedad Española de Educación Médica (SEDEM). Sesión Temática 3: Las Facultades de Medicina y el Proceso de Convergencia Europea. Educación Médica, 6,3:2. 2003,

36. XVII Congreso de la Sociedad Española de Educación Médica (SEDEM). Educación Médica Internacional,; 8, 3, 2005.

37. Sociedad Española de Educación Médica (SEDEM). Declaración del Lazareto de Mahón. Evaluación de las competencias profesionales en el pregrado. Educación Médica, 7 : 103105,2004 
38. Sociedad Española de Educación Médica (SEDEM), Associació Catalana d'Educació Mèdica (ACEM), Sociedad de Educación Médica de Euskadi (SEMDE), Sociedad Aragonesa de Educación Médica (SADEM). Recomendaciones para un nuevo proceso de reforma curricular en las Facultades de Medicina Españolas. Educación Médica, 8:3-7, 2005

39. Conferencia Nacional de Decanos de Facultades de Medicina Españolas. Libro Blanco del Título de Grado en Medicina. http://www.aneca.es/modal_eval/conver_docs-titulos.html

40. Facultades de Medicina de la UB, UAB, UDL y URV y AQU Catalunya. Competencias profesionales de los Licenciados en Medicina, formados en las Universidades Catalanas, 2004. www.aqu-catalunya.org/scripts/web/ llistat.asp?cid=Esp\&cat= pubbl\&id=2409\&res $=10$

41. Ficha Técnica de Propuesta de Título Universitario de las Enseñanzas de Medicina, con el fin de adaptarse al Espacio Europeo de Educación Superior. Ministerio de Educación y Ciencia, 2006.

42. Sociedad Española de Educación Médica (SEDEM). Informe sobre la ficha técnica de propuesta de título universitario de las enseñanzas de Medicina. Junio 2006. http://www.sedem.org

43. Alfonso Roca, MT. El aprendizaje de la Medicina en la universidad de Castilla La Mancha. Una experiencia educativa basada en el aprendizaje de ls competencias profesionales. Educación Médica vol 8, sup.2, 9-12, 2005.

44. Institute for Internacional Medical Education: Global Minimum Essential Requeriments in Medical Education.Educación Médica vol. 6, Supl. 2, 2003

45. Oriol-Bosch, Albert.. El curriculum de Medicina. Reflexiones no publicadas. 2005

46. Pales J, Cardellach F, Estrach MT, Gomar C, Gual A, Pons F \& Bombi JA.- Defining the Learning Outcomes of Graduates from the Medical School at the University of Barcelona. Catalonia, Spain). Medical Teacher vol. 26 (3), pags 239-243, 2004

47. Porcel JM, Esquerda JE, Pena M \& Viñas J:"Competències Professionals a assolir durant el Període de formació de pregrau a la Facultat de Medicina,Universitat de Lleida" Edicions de la Universitat de Lleida, 2004.

48. Simpson JG, Furnace J, Crosby J, Cumming AD, Evans PA, Friedman Ben David M, Harden RM, Lloyd D, McKenzie H, McLachlan JC, McPhate JF, Percy-Robb IW, Pherson SG : The Scottish Doctor. Learning outcomes for the medical undergraduate in Scotland: a foundation for competent and reflective practitioners. Med Reach vol. 24, 136-143, 2002

49. Harden, R.M. and Friedman, M. From competency to metacompetency: a model for the specification of learning outcomes, AMEE Medical Education Guide $n^{\circ} 14$ : Outcome-based education: Part 5- Medical Teacher, 21, 6), 546-552. 1999.
50. Harden, RM. Ten questions to ask when planning a course or curriculum. Medical Education, 20: 356-365, 1986

51. Palés, J. Planificar un curriculum o un programa formativo. Educación Médica 9(2): 59-65, 2006

52. Barrows, HS. y Tamblyn, E.M. (1980). Problem based-learning. An approach to medical education. New York Springer Publications.

53. Carreras. J. (1999). Elementos de estrategia pedagógica e implicaciones metodológicas. Educación Médica, vol 2, $\mathrm{n}^{\circ}$ 1, 2-7.

54. Roma, J. La enseñanza basada en problemas y la enseñanza en grupo: una alternativa para estudiantes, residentes y profesionales. Educación Médica, 2(3), 104-106, 1999.

55. Oriol-Bosch, Albert, (2004). Simulacions. Reflexiones no publicadas.

56. Palés J and Gomar. C, (2006). Implementation of a centralised skills laboratory at the medical school of the Barcelona University. AMEE Annual Meeting, Genoa. (Italy)

57. Smith, Barry, (2006). From simulation to reality- breaking down the barriers. The Clinical Teacher, 3, 112-117.

58. Van Dalen, J. (1990). Skills lab- a centre for a training of skills. In: Van de Leuten, C, Wijen W, eds. Problem based learningperspectives from the Maastricht experience. Amsterdam: Thesis

59. Ziv,A., Wolpe,P.R., Small,S.D., \& Glick,S. (2004). SimulationBased Medical Education : an Ethical Imperative. Academic Medicina vol. 78, 8, 783-788.

60. Shunmway, JM and Harden, RM. The assessment of learning outcomes dor the competent and reflective physician. AMEE Medical Education Guide $\mathrm{n}^{\circ}$ 25, 2003.

61. Blay, C. (1998). Evaluación clínica objetiva y estructurada: ECOE. Educación Médica, vol 1, n 1, 13-16.

62. Bombí, JA. La enseñanza de la Medicina en las Facul-tades españolas. Educación Médica, 5(1): 22-26, 2005

63. Wojtczak, A. Professionalism, a global headache. Educación Médica, 9(3), 2006, en prensa

64. General Medical Council. Good Medical Practice.

(http//www.gmc-uk.org/Standard/good.htm)

65. Oriol-Bosch, A. El nuevo profesionalismo. El médico deseado para el Siglo XXI. VIII Ateneo de Bioética. Madrid 25 de Octubre de 2006

66. XVI Congreso de la Sociedad Española de Educación Médica, Madrid 2003. Conclusiones. Educación Médica 7(4), 2003.

67. WFME y OMS. Guía de la OMS / WFME para la acreditación de la formación médica de grado. Educación Médica, 9(3) en prensa, 2006 